\title{
Pneumonia Induced Childhood Erythema Multiforme Minor: A Case Report
}

\author{
Putri Dahliana, Khairuddin Djawad*iD, Farida Tabri \\ Department of Dermatology and Venereology, Hasanuddin University, Makassar, South Sulawesi, Indonesia
}

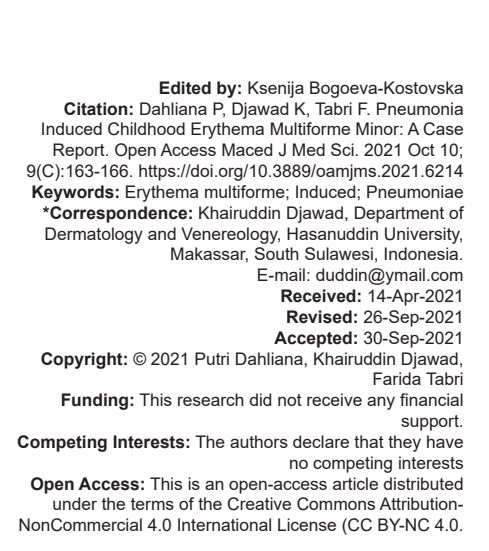

\section{Abstract}

Erythema multiforme (EM) is an acute mucocutaneous syndrome mediated by hypersensitivity reactions. Symptoms are often mild and self-limiting but have a high recurrency rate. The disease is more prevalent in adolescents and adults, with incidence in childhood often precipitated by infection of herpes simplex virus type 1. However, EM induced by infection of Mycoplasma pneumonia is a rare occurrence. This paper reports a case of pneumonia induced EM in a 5-year-old child with a typical clinical feature supported by laboratory and chest X-ray features that were confirmed with histopathology. The patient showed significant clinical improvements after antibiotic therapy.

\section{Introduction}

Erythema multiforme (EM) is an acute, mucocutaneous self-limited disease characterized by the appearance of symmetrical red papules that develop into typical, characteristic target lesions with concentric color variations, or atypical lesions. Mucosal lesions are found in more than $70 \%$ of cases and are usually confined to the oral area with predilection areas on both lips, gums, and ventral areas of the tongue. Erythema indicates discoloration of the skin caused by dilated blood vessels, especially in the dermis of the pars reticular and pars papillary. In severe cases, constitutional symptoms and visceral lesions can occur [1], [2].

EM prevalence is rare at $<1 \%$ worldwide, of which one fifth of cases occur in children, with a ratio of 5:1 between males and females [1], [2]. In most cases, EM in children is precipitated by herpes simplex virus (HSV) type 1, whereas HSV type 2 is more common in young adults. Other viral infections such as varicella zoster virus and Epstein-Barr virus can also trigger EM in children [3]. Cases induced by Mycoplasma pneumoniae and other bacterial infections have also been reported [4].
Diagnosis of EM is often clinically made as there is no specific diagnostic test for EM. Histopathological examination can also help to rule out other diagnoses. The appearance of pulmonary symptoms such as coughing and tightness can further support the possibility of infections. Any suspicion of M. pneumoniae as a cause should be systematically sought in children. M. pneumoniae in children account for $2-10 \%$ of complicated EM cases, of $2 / 3$ will have mucosal involvement. It is recommended to perform a systematic chest X-ray and to bacteriological assessment, if possible by PCR for confirmation of M. pneumonia [5].

\section{Case Report}

A 5-year-old boy came to the emergency room with the chief complaint of erythematous rash across the body accompanied with high fever 3 days prior. Previously, the patient went to the local public health-care center, and received paracetamol, antihistamines, and dexamethasone. After 2 days of treatment, the patient experienced no improvements. 
The patient had a history of coughing, flu, and sore throat around 2 weeks before the appearance of the rash.

Physical examination found that vital signs were within normal limit. Dermatological examination found generalized erythematous macules, patches, and plaques along with targeted lesions with central erythema, pale peripheries, and erythematous circles or halos of various sizes (Figure 1). There were no abnormalities found on the mucosa. Based on the typical clinical presentation, the patient was initially diagnosed with EM, with a differential diagnosis of urticaria multiforme.

Further histopathological, complete blood count, HSV serology, and chest X-ray tests were performed to find the underlying etiology of EM. Histopathological examination showed hyperkeratosis, basal cell vacuolar degeneration microfocus, and spongiosis. In the upper dermis, there was mild perivascular inflammation of lymphocytes, which supported EM (Figure 2). Complete blood count showed leukocytosis (18.800/ul) and an increased in leukocyte sedimentation rate $(21 \mathrm{~mm} / \mathrm{h})$. Other parameters were within normal limits. Serological tests of IgG and IgM anti-HSV 1 and 2 were negative using the ELISA method. Chest X-ray found that the appearance of infiltrates in the right lower lung field with the impression of community acquired pneumonia (Figure 3).

The final diagnosis of this case was pneumonia induced EM. The patient was treated with oral erythromycin $250 \mathrm{mg} 3$ times daily, oral cetirizine $5 \mathrm{mg} /$ daily, and topical corticosteroids of desoximethasone. After 2 weeks of treatment, the patient showed significant clinical improvements and was discharged from the hospital (Figure 4).

\section{Discussion}

EM is an acute mucocutaneous condition, associated with cutaneous eruptions that can be induced by various precipitating factors with immunological basis, often associated with a type-IV delayed-type hypersensitivity reaction [6]. The clinical manifestation of the disease is characterized by the onset of symmetrical red papules that develop into a typical target lesion of concentric or atypical color variations. Lesions appear within $24 \mathrm{~h}$ and fully develop within $72 \mathrm{~h}$. EM is classified into two major classifications based on the degree of mucous membrane involved. In the minor variant $(E M m)$ patients have a single or no mucosal involvement, whereas in the major variant of EM (EMM) involvement of multiple mucous membranes is found, along with systemic symptoms such as fever and arthralgia. Apart from these two major classifications, EM can also be divided into other subtypes, namely, atypical EMM, mucosal EMM, and persistent EM. There is a close relationship between the different subtypes and their etiology [2]. EM can occur across a wide spectrum of ages but is more common in adolescents and adults. Occurrence in children is rare [1], [2]. Typical EM lesions present as regular, circular, and raised erythematous papules or plaques, which past 1 week or longer that measures between a few millimeters to $3 \mathrm{~cm}$ and can continue to enlarge in 24-48 h. Although the peripheral area remains erythematous and edematous, hyperpigmentation can occur in the center of the lesion along with decreased inflammation, resulting in a concentric circle of color. Occasionally, development of tense bullae, vesicle, or necrosis can occur on the central of the lesion. The result is a classic target lesion or iris lesion [2], [3].

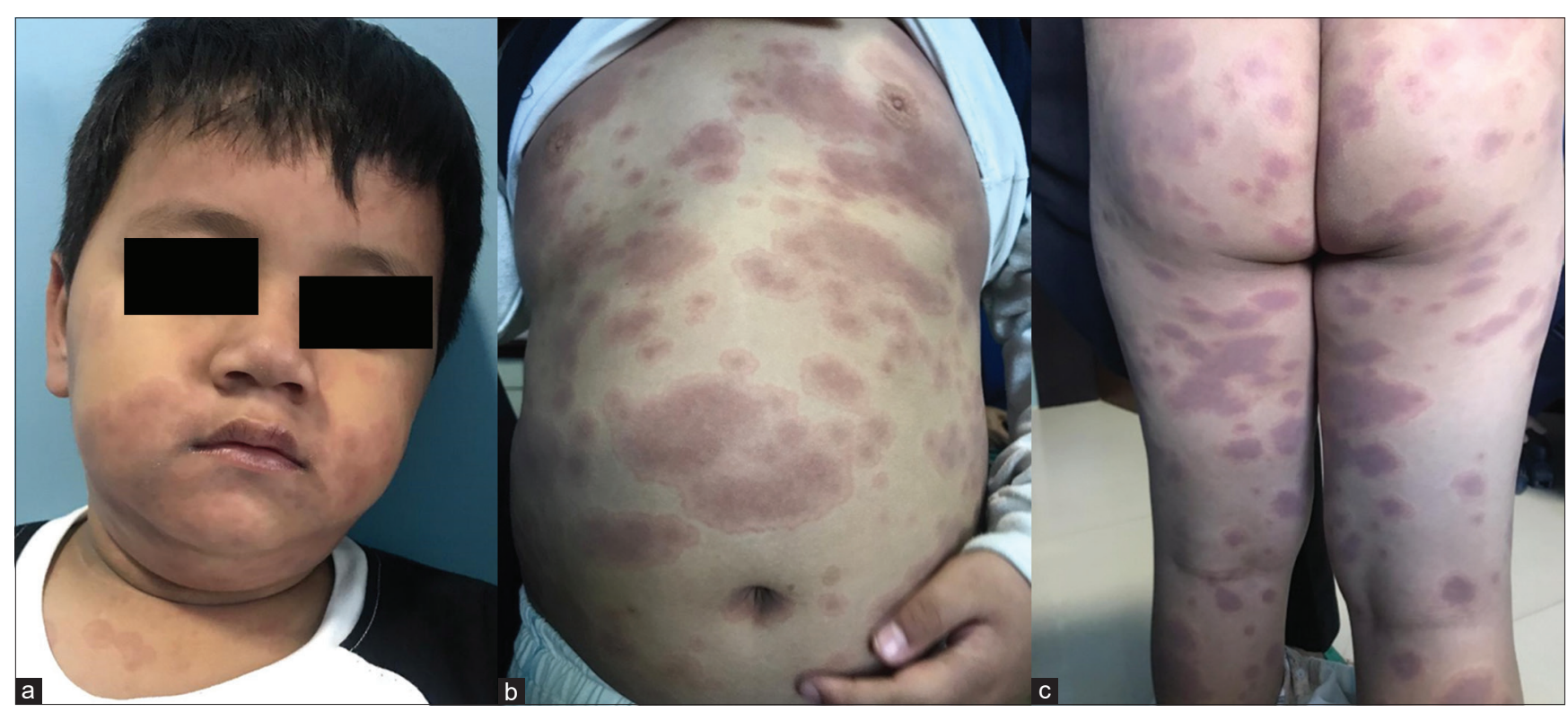

Figure 1: (a-c) Generalized target lesions 


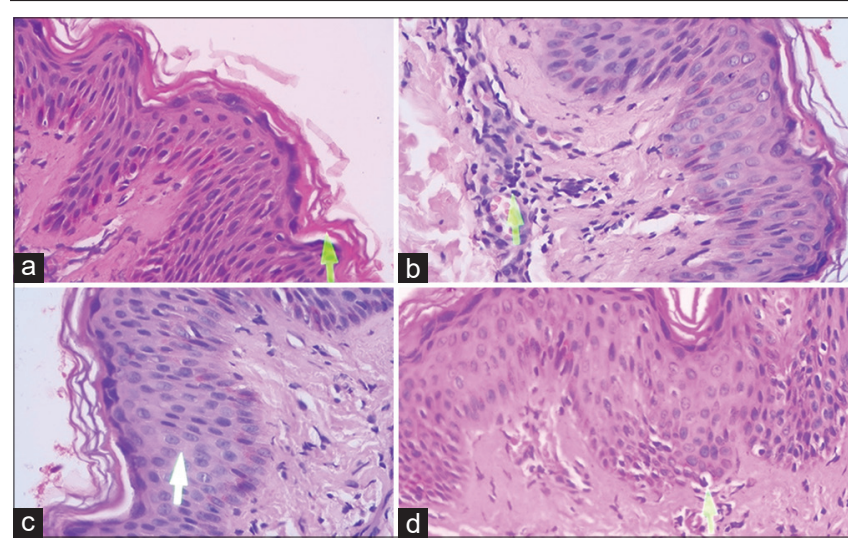

Figure 2: Hyperkeratosis (a), lymphocytes on the upper dermis (b), spongiosis (c) and basal cell vacuole degeneration (d)

Histopathological examination in this patient found lymphocyte accumulation in the dermalepidermal accompanied by exocytosis in the epidermis, lymphocyte adhesions, the spread of necrotic keratinocytes (satellite cell necrosis), spongiosis, vacuolar degeneration of basal cells, and characteristic to EM [1], [2], [3].

About $90 \%$ of cases of EM in children are triggered by infections. HSV type 1 is the most common cause in childhood, whereas HSV type 2 is more frequently associated with EM in adolescents and adults [2], [3]. In this case, serologic tests for IgG and IgM of HSV type 1 and type 2 were negative. The patient had respiratory symptoms in the form of cough and flu, and chest $\mathrm{X}$-ray showed infiltrates on the lower right lung suggestive of pneumonia. In addition, children are known to be more prone to pneumonia [2], [7]. Minor EM was the final diagnosis of the patient as there was no mucosal involvement found. Interestingly, literatures stated that pneumonia is often found in EM major [7] [8].

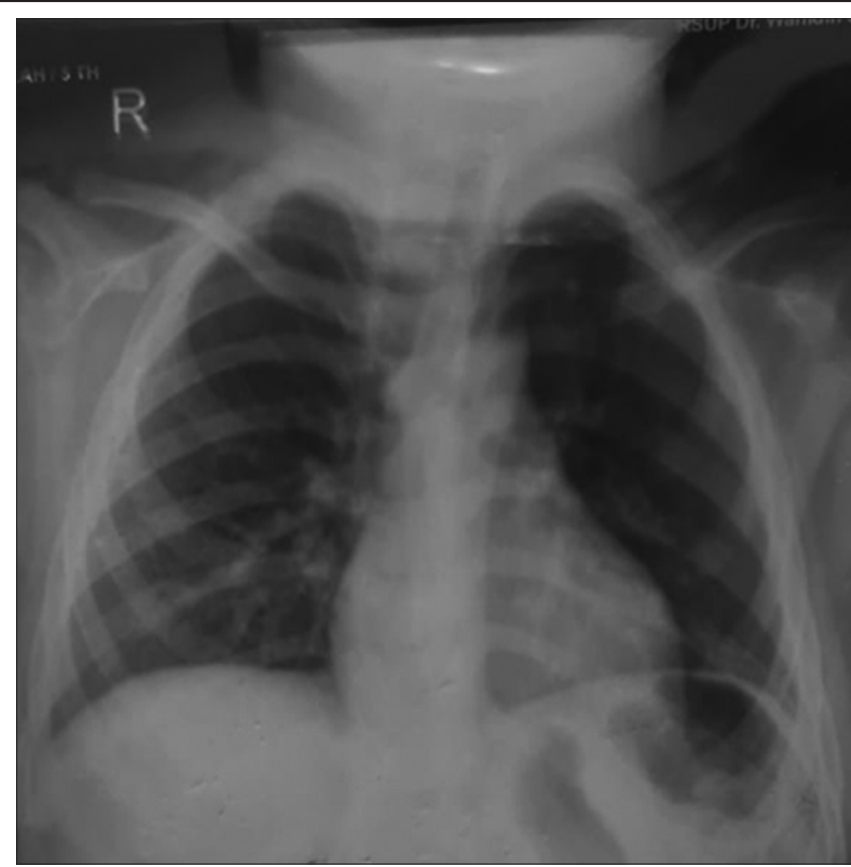

Figure 3: Chest $X$-ray showed infiltrates on the lower right lung

One of the differential diagnoses for this case is urticaria multiforme. Even though both diseases have similar clinical appearances, lesions of EM pasts $>24 \mathrm{~h}$, whereas in urticaria multiforme, in $72 \%$ of cases, lesions will resolve within $24 \mathrm{~h}$ of initial onset [2], [9] Another possible differential diagnosis is $M$. pneumoniae induced rash and mucositis (MIRM). However, even though the suspected pathogen is $M$. pneumoniae, the patient experienced no mucosal involvement with generalized rash, whereas in MIRM lesions usually only affect $10 \%$ of BSA with at least two mucosal involvements [2], [9].

The use of systemic steroids is recommended as it can suppress cytokine, chemokine responses, and $T$ cell function, which results in decreased adhesion of

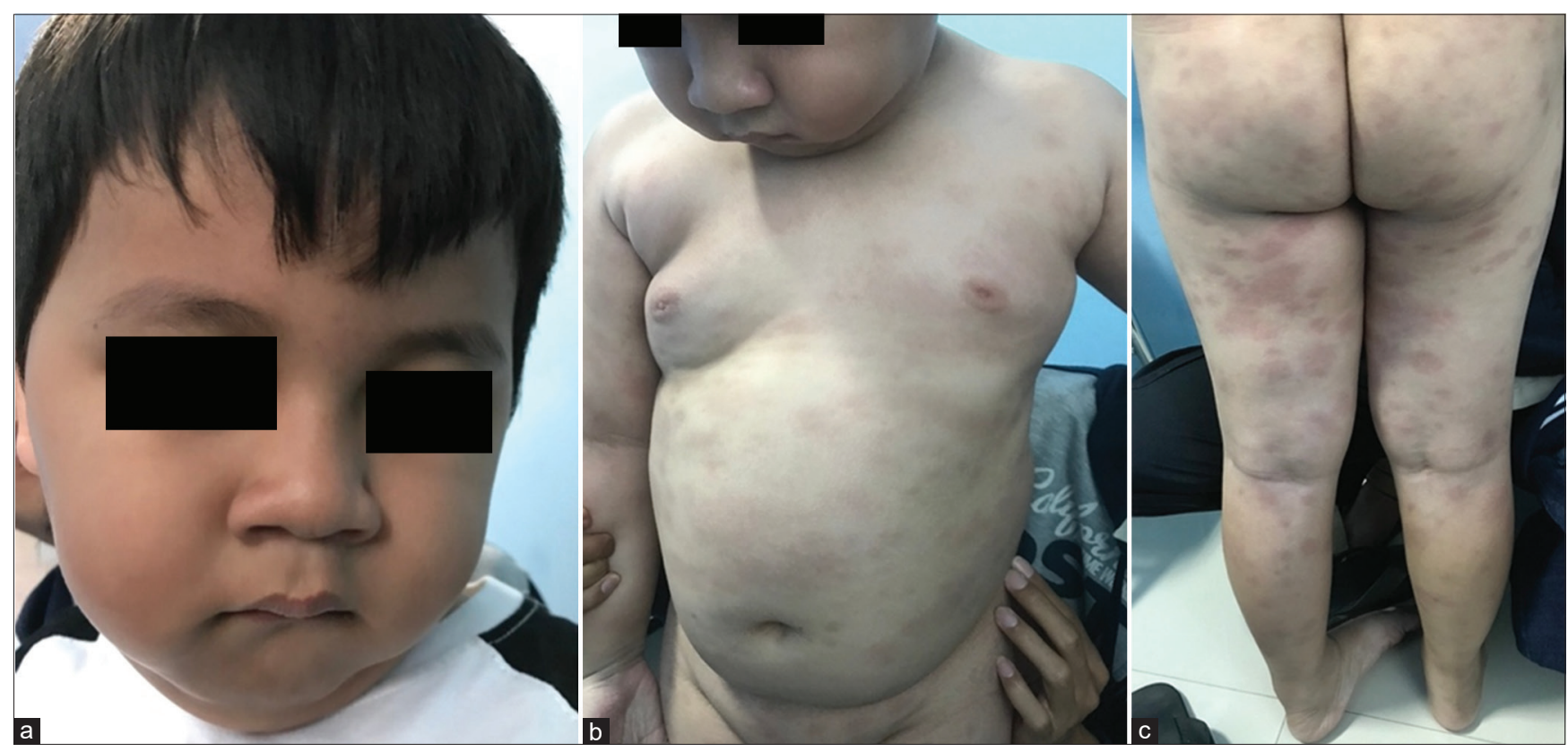

Figure 4: (a-c) Resolution of lesions after antibiotic therapy 
inflammatory molecules to the endothelium of blood vessels [10]. The use of systemic corticosteroids can also shorten the duration of fever and eruption and should be considered for use in cases of EMM. In child EMM, systemic corticosteroids can be given at a dose of $0.5-1 \mathrm{mg} / \mathrm{kg} / \mathrm{day}$ of prednisone for 3 days or 1-2 mg/ kg/day of methylprednisolone [2]. In our case, we applied topical desoximethasone as it also proved to reduce inflammation, and help to constrict capillaries reducing erythema [2].

The first objective of therapy in EM is to eliminate precipitating factors. If the EM is caused by HSV, oral acyclovir is recommended especially in children. In cases caused by M. pneumoniae and giving symptoms, macrolide or tetracycline antibiotics are the treatment options [1], [10]. Erythromycin can be an option in children especially those younger than 8 years [2]. In our case, the combination therapy of oral antibiotics and topical corticosteroids in conjunction with oral antihistamine provided great result with reduction of lesions within 2 weeks of treatment.

\section{Conclusion}

EM is a mucocutaneous eruption with a characteristic clinical appearance of a target lesion. Even though self-limiting, the disease has a high recurrence rate. Cases of EM in children are rare, and investigating the underlying etiology is a key in treating the disease. In our case, treating the underlying etiology of pneumonia provided great outcome.

\section{References}

1. Mockenhaupt M, Roujeau JC. Erythema multiforme. In Fitzpatrick's Dermatology in General Medicine. $9^{\text {th }}$ ed. New York: McGraw-Hill Education; 2019.

2. Wolfram HC. Erythema multiforme, Stevens-Johnson syndrome, and toxic epidermal necrolysis. In: Fourth edition dermatology Bolognia. $4^{\text {th }}$ ed. New York: Elsevier Inc.; 2018. https://doi. org/10.1016/b978-0-7020-3093-2.10003-2

3. Paller A. The hipersensitivity syndrome. In: Hurwitz Clinical Pediatric Dermatology. $5^{\text {th }}$ ed. Canada: Elsevier; 2015.

4. Imashuku S, Kudo N. Chlamydia pneumonia infectionassociated erythema multiforme. Pediatr Rep. 2013;5(2):35-7. https://doi.org/10.4081/pr.2013.e9 PMid:23904964

5. Wissem H, Tele B. Erythema Multiforme. New York: StatPearls Publishing LLC; 2019.

6. Malcolm R, Rino C. Reactive in ammatory erythemas. In: Rook's Textbook of Dermatology. $4^{\text {th }}$ ed. United Kingdom: John Wiley and Son; 2016

7. Langley A, Anooshiravani N, Kwan S, Zeller J, Pope E. Erythema multiforme in children and mycoplasma pneumonia etiology. J Cutan Med Surg. 2016;20(5):453-7. https://doi. org/10.1177/1203475416639018 PMid:26976263

8. Bau JT, Cooper C. Erythema multiforme major associated with Mycoplasma pneumoniae infection. CMAJ. 2019;191(43):E1195. https://doi.org/10.1503/cmaj.190850

PMid:31659061

9. Siedner WY, Gross I, David A, Reif S, Molho P. Pediatric erythema multiforme: Epidemiological, clinical, and laboratory characteristics. Acta Derm Venereol. 2017;97(4):489-92. https:// doi.org/10.2340/00015555-2569 PMid:27868144

10. Chan M, Goldman R. Erythema multiforme in children: The steroid debate. Can Fam Physician. 2013;59(6):635-6 PMid:23766045 